Santa Ana de Coro, Venezuela

\title{
Las habilidades resuntivas de ensayos académicos expositivos en estudiantes de la Universidad Nacional Experimental Francisco de Miranda
}

\section{Resunctive skills of academic expository essays in students of the National Experimental University Francisco de Miranda}

\author{
Yovaida Dovale \\ yovaidaalejandra@gmail.com \\ Universidad Nacional Experimental Francisco de Miranda, Coro \\ Venezuela
}

Recibido: 15 de octubre de 2017

Aprobado: 15 de noviembre de 2017

\begin{abstract}
RESUMEN
Resumir un ensayo académico expositivo es un proceso constructivo demandante que requiere el entendimiento de información textual implícita y explícita del texto a ser resumido, así como la aplicación de macroestrategias, tales como la de selecciónsupresión y generalización (Van Dijk y Kintsch, 1983). Aunque a los estudiantes les es solicitado frecuentemente resumir un texto académico, ellos escasas veces son entrenados en información procedimental acerca de cómo se lleva a cabo un resumen. Este estudio persigue evaluar las habilidades resuntivas de ensayos académicos expositivos mostradas por un grupo de doce (12) estudiantes de Educación de la UNEFM, a quienes les fue solicitado leer cuidadosamente un ensayo académico expositivo de nueve párrafos y elaborar un resumen de 25 líneas de su contenido. Los resultados revelaron habilidades resuntivas pobres de las estudiantes, evidenciadas por la inadecuada selección de la información relevante del ensayo, la preservación de piezas de información irrelevante y la inclusión de nueva información y opiniones personales. Además, los resúmenes analizados exhibieron una falla superestructural, ya que en muchos de ellos $(90 \%)$ el tópico fue directamente desarrollado sin ninguna introducción o cierre. El uso inadecuado de elementos microestructurales fue observado principalmente a nivel cohesivo. Se sugiere entrenar a los estudiantes y profesores en el empleo adecuado de macroestrategias resuntivas y en las características discursivas que identifican diferentes géneros textuales, de manera que los estudiantes puedan producir muestras resuntivas apropiadas como producto de comprensión textual.
\end{abstract}

Descriptores: Ensayo; Macroestrategias; Resumen; Estudiantes; Habilidades.

\section{ABSTRACT}

Summarizing an expository academic essay is a demanding constructive process that requires the understanding of explicit an implicit textual information of the text being summarized as well as the application of macrostrategies such as selection-supression 
and generalization (Van Dijk y Kintsch, 1983). Though students are frequently asked to summarize academic texts, they are rarely provided with conceptual and procedural information about how this is to be done. This study aims at evaluating the summarizing abilities of expository academic essays showed by a sample of twelve (12) students of Education at UNEFM who were asked to read carefully a nine paragraph academic expository essay and instructed to write a 25 lines summary of its content. Results revealed students' poor summarizing abilities showed by the inadequate selection of relevant information, preservation of irrelevant pieces of information, inclusion of new information and personal opinions. Besides the summaries analyzed exhibited a default superstructure, since in most of them (90\%) the topic was directly developed without any introduction or close an non adequate use of microstructural elements was observed, mainly at the cohesive level. It is suggested to train students and teachers on the adequate use of summarizing macrostrategies and on the discursive features which identify different textual genres, so that students can produce suitable summary samples as textual comprehension products.

Descriptors: Essay; Macrostrategies; Summary; Students; Abilities.

\section{INTRODUCCIÓN}

El resumen de textos parece ser una tarea cotidiana en las aulas universitarias con el propósito de evaluar la comprensión de la lectura. La habilidad para extraer información textual relevante y agruparla en porciones dotadas de cohesión y organización representa para docentes y estudiantes un indicador irrefutable de comprensión de textos (Álvarez, 1996), especialmente de ensayos académicos, género textual de común lectura en esta población. Sin embargo, es poco lo que los estudiantes conocen acerca de las estrategias cognitivas que operan para elaborar un resumen que pueda ser considerado adecuado. No se evidencian actividades pedagógicas que permitan el desarrollo de habilidades de reformulación, en este caso, de tipo resuntivo, en los estudiantes universitarios. Tal hecho es lo que revelan investigaciones llevadas a cabo por Bracho (2008) y Jordán (2009) al concluir que a sus muestras les era difícil plasmar por escrito su comprensión sobre el texto que se les había provisto, formándose así interrogantes que giraban en torno a saber si estas dificultades guardaban relación con la comprensión del texto en sí, o con la existencia de problemas para replantear la información leída.

En base a ello, el presente estudio pretendió evaluar las habilidades resuntivas de ensayos académicos expositivos por parte de los estudiantes de la Universidad 
Nacional Experimental Francisco de Miranda (UNEFM) a partir de la lectura de un ensayo académico de tipo expositivo para analizar su macroestructura y las macroestrategias empleadas por los mismos. Se buscó con ello conocer las habilidades resuntivas de esta población, a los fines de formular sugerencias tendentes a subsanar el problema observado con base en información confiable producto de la aproximación empírica a los datos.

\section{DESARROLLO}

La escritura representa una forma de comunicación diferida en el tiempo y, por ello, desarrollar en el estudiante las estrategias necesarias para alcanzar los niveles literal, inferencial y crítico de comprensión lectora representa hoy en día una de las tareas más demandantes para docentes y estudiantes de todos los niveles del sistema de educación venezolano (DaCosta, 2008). Por tanto, conscientes de esa exigencia y de las limitaciones que como lectores arrastran los bachilleres de nuevo ingreso, el Programa de Educación de la Universidad Nacional Experimental Francisco de Miranda (UNEFM), en los primeros semestres de sus planes de estudios de sus diversas menciones ofrece unidades curriculares tales como: Lectura y Expresión I y II, Lenguaje y Comunicación y Técnicas de Estudio y Documentación, las cuales forman parte del eje de formación instrumental y están destinadas a desarrollar en los estudiantes las habilidades y estrategias académicas que les apoyen en el estudio de unidades curriculares más especializadas; Por ello, entre sus objetivos contemplan, total o parcialmente, el desarrollo o consolidación de los niveles de comprensión lectora en estos estudiantes. Entre las actividades empleadas con mayor frecuencia para lograr estos objetivos se encuentra la lectura de ensayos que posteriormente deben ser resumidos.

A pesar de ese esfuerzo curricular sistemático, de acuerdo a frecuentes planteamientos de docentes de las unidades curriculares antes mencionadas en reuniones del Departamento de Idiomas de esta casa de estudios, aún después de haber aprobado estas asignaturas, los estudiantes parecen seguir mostrando dificultades al momento de desarrollar resúmenes de ensayos previamente leídos; parece ser que estas dificultades son más frecuentes en aquellos estudiantes que 
cursan estudios bajo la modalidad de Aprendizaje Dialógico Interactivo. Este problema incide significativamente en el rendimiento académico, toda vez que, tanto docentes de estas asignaturas como los de otras correspondientes a ejes curriculares como el pedagógico o el de formación especializada, solicitan la elaboración de resúmenes como parte de las actividades cotidianas de aprendizaje y evaluación.

Por su parte, el ensayo académico expositivo constituye un género con características difíciles de precisar, ya que puede adoptar una orientación expositiva o argumentativa (Fumero, 1997) o combinar ambas tendencias con predominio de una sobre la otra (García de Díaz, 2008). Cada una de estas variantes puede hacer uso de organizaciones, tales como problema-solución, causa-efecto, comparación-contraste, entre otras. Esta amplia variabilidad de funciones y estructuras organizativas agrega mayor complejidad a la comprensión de ensayos académicos y, por tanto, genera aún mayores dificultades al estudiantado para elaborar muestras resuntivas.

La escasa competencia discursiva que parece mostrar los estudiantes universitarios para elaborar resúmenes partiendo de este género textual compromete el perfil del egresado que la institución se propone formar. Además, la presencia de estas deficiencias podría causar problemas a los egresados en su futuro ejercicio profesional, debido a que en algún momento deberán enseñar a sus alumnos a resumir.

La memoria a corto plazo (MCP) presenta limitaciones para almacenar toda la información nueva que, a lo largo de su vida académica, un estudiante debe procesar. Para ello, requiere de mecanismos que permitan sintetizarla y vincularla a la información ya conocida, a objeto de que pueda ser almacenada en la memoria a largo plazo (MLP) (León, 1996 y García, 2006). De no ser sintetizada esta nueva información, la MCP del estudiante colapsaría y, por tanto, no podría comprender inferencial ni críticamente lo que lee, sino limitarse a una comprensión literal de textos que apenas le permitiría repetir literalmente lo que ha leído (Smith, 1985). De allí que el desarrollo de habilidades resuntivas tiene gran importancia en la formación del estudiante universitario.

Al respecto, para resumir o reformular resuntivamente un texto, se requiere emplear eficientemente la síntesis o la paráfrasis como estrategias de reformulación (Van Dijk 
y Kinstch, 1983). Para ello es necesario que quien construye el resumen haya comprendido el texto fuente, lo que implica relacionar la información nueva del texto con los conocimientos previos y así integrarlo a los esquemas cognitivos (Arnoux, Noguera y Silvestre, 2006). Este no parece ser el caso de la población estudiantil descrita, ya que de acuerdo al testimonio de los docentes de las asignaturas antes mencionadas, los estudiantes exhiben limitaciones en la selección de información textual relevante y dificultades para organizarla en sus muestras resuntivas.

El problema que se estudia en esta investigación se concreta a dar respuesta a la siguiente interrogante: ¿Cuál es la calidad de las habilidades resuntivas exhibidas por los estudiantes del Programa de Educación de la Universidad Nacional Experimental Francisco de Miranda (UNEFM) al momento de elaborar resúmenes de ensayos académicos expositivos previamente leídos?

Para dar respuesta a esta interrogante, se plantea como objetivo general Evaluar las habilidades resuntivas de los estudiantes del Programa Educación de la Universidad Nacional Experimental Francisco de Miranda (UNEFM) al elaborar resúmenes de ensayos académicos expositivos. Para ello, se pretende cumplir con los siguientes objetivos específicos: Identificar los rasgos Macroestructurales de los resúmenes de ensayos académicos expositivos compuestos por estos estudiantes, explorar el uso de las macroestrategias discursivas empleadas por tales estudiantes para resumir ensayos académicos expositivos y establecer las relaciones entre la macroestructura de los resúmenes elaborados y el uso de las macroestrategias empleadas en su elaboración.

Para lograr el objetivo que persiguió esta investigación se revisaron las nociones correspondientes a la concepción del ensayo académico con tendencia expositiva como género discursivo, en términos de: propósito del autor (dar a conocer un tema, estimular el análisis y la reflexión y modificar el estado epistémico del lector potencial) (Calsamiglia y Tusón, 1999); estructura organizativa, conformada por una introducción (tópico y objetivo del ensayo), un desarrollo (presentación de la información en términos de características, funciones, aplicabilidad de un objeto, concepto) y cierre (idea conclusiva y resuntiva del tema tratado) (Asnar, Cross y Quintana, 1991); estrategias discursivas típicas de este género textual (definición, clasificación, 
ejemplificación y descripción, para ayudar al lector a la asimilación de nuevos significados y en la integración de los mismos con su conocimiento previo) (Álvarez, 1996 en Calsamiglia y Tusón, 1999); organización de los párrafos (causa- efecto, comparación-contraste, de ilustración, cronológicos, descriptivos) (Álvarez, 1996); y progresión informativa típica (lineal, de tema constante, de tema derivado) (Calsamiglia y Tusón, 1999).

A su vez, el ensayo académico expositivo fue estudiado a través del modelo macroestructural de Van Dijk y Kintsch (1983) que postula que en la comprensión y producción de textos subyacen proposiciones (significado básico expresado por una oración, aquello que se nombra o se predica) que se encuentran en oraciones y se entrelazan con otras a través de redes semánticas en los textos, para formar las macroproposiciones (Richards, Platt y Platt, 1992). Este conjunto de relaciones se establece a través de una estructura organizativa jerarquizada, que incluye tres niveles interrelacionados en el texto: la macroestructura (conjunto de proposiciones que sintetizan el significado global del texto, para preservar su relevancia y temática), la microestructura (conjunto de proposiciones que rescatan significados complementarios y que se relacionan localmente) y la superestructura (nivel que determina la forma en la que está organizado el texto) (Calsamiglia y Tusón, 1999).

Por otra parte, el modelo secuencial de Adam (1990) permitió complementar el análisis del ensayo académico expositivo como género a través de la descripción de las secuencias textuales (descriptiva, argumentativa y explicativa) que en estas producciones escritas pueden converger y que, por tanto, complejizan su comprensión y, por ende, el rescate de la información relevante al momento de resumirlas.

Finalmente, se estudió el modelo de Van Dijk y Kintsch (1983) como elemento coadyuvante en la descripción del proceso mediante el cual se construye una comprensión profunda y sintetizada de un texto, a través de la descripción de las macroestrategias de lectura o de reformulación, que permiten reducir y organizar la información explícita e implícita del texto, facilitando al lector la comprensión de su contenido, reduciendo las proposiciones de la microestructura y manteniendo las de la macroestructura textual, por lo que preservan las ideas principales de la muestra escrita. Estas estrategias son la supresión (eliminación de proposiciones que no son 
necesarias para interpretar proposiciones posteriores), la generalización (sustitución de una proposición por otra más general que organice y sintetice el contenido de varias) y la construcción (sustitución de un conjunto de proposiciones por una más simple, que, reformulada, de cuenta de todas ellas) (Van Dijk y Kintsch, 1983 en Calsamiglia y Tusón, 1999). Durante el empleo de estas estrategias los niveles de comprensión literal e inferencial se encuentran estrechamente vinculados, ya que para lograr extraer información textual implícita (comprensión inferencial) resulta necesario poder reconocer información textual explícita (comprensión literal). Es decir, no se puede suprimir, generalizar o construir información relevante si no se es capaz de reconocer o evocar información textual irrelevante.

Por tanto, no se puede resumir aquello que no se ha comprendido, por lo menos, a nivel inferencial. Además, no se puede resumir si no se es capaz de sintetizar y la síntesis se expresa, justamente, a través de la aplicación de las macroestrategias de reformulación descritas que permiten reducir el texto a sus rasgos macroestructurales o significados globales fundamentales. La síntesis es el mecanismo de abstracción a través del cual se obtiene la información relevante de un texto escrito y su producto concreto es el resumen (Fraca, 2001).

Ahora bien, desde el punto de vista metodológico, la presente investigación constituyó un estudio de campo de naturaleza descriptiva, transeccional, evaluativa y no experimental, considerando que los datos se obtuvieron directamente de la realidad, en un tiempo y lugar específicos y que no se llevó a cabo ningún procedimiento para manipular las variables en estudio (Arias, 2009).

La investigación tuvo como centro de estudio a la población correspondiente a los 19 estudiantes del segundo semestre de las menciones de Inglés (9), Lengua, Literatura y Latín (10) de la Modalidad de Aprendizaje Dialógico Interactivo (ADI) UNEFM. Estos estudiantes eran todos cursantes regulares de las asignaturas Lectura y Expresión II y Lenguaje y Comunicación, respectivamente. La población fue seleccionada considerando que es justamente en estas unidades curriculares en donde se presenta el problema descrito en líneas anteriores. Considerando que el tamaño de la población así lo permitía, se trabajó con todo el grupo, por lo que la muestra se calificó como intencional y no probabilística (Arias, 2009). No obstante, al momento de la aplicación 
del instrumento se contó con la asistencia de 5 estudiantes de la Mención Lengua, Literatura y Latín y 7 de la Mención Inglés, por lo que la muestra quedó reducida a 12 estudiantes.

La técnica de investigación empleada fue la observación directa y no participante, sistematizada por medio de una prueba de ejecución en la cual se le instruía a los participantes, mediante dos enunciados imperativos a ejecutar acciones (Hernández, Fernández y Baptista, 1998): leer cuidadosamente un ensayo académico y elaborar un resumen del mismo en un máximo de 25 líneas de extensión. El cuestionario estuvo constituido por dos partes. En la primera, se pedía a los sujetos leer un ensayo académico expositivo titulado "La Enseñanza Tradicional vs. La Enseñanza Virtual", cuyo contenido se consideró familiar para los estudiantes, ya que cursan estudios bajo una modalidad dialógica e interactiva. El contenido del mismo giraba en torno a las ventajas y desventajas de los modelos de enseñanza tradicional y virtual cada uno de los cuales era descrito, explicado y criticado para finalmente, ofrecer un cierre señalando que la mejor opción era combinar ambos enfoques de forma estratégica. La versión original de este ensayo fue analizada previamente por la investigadora para determinar sus características macro, super y micro estructurales. Dicho instrumento fue validado por cuatro expertos magisters en la Enseñanza de la Lectura y la Escritura, adscritos al Departamento de Idiomas de la UNEFM. No fue requerido el cálculo de la confiabilidad, considerando que los instrumentos diseñados constituyen herramientas no convencionales de recolección de información (Ruíz, 2002).

Se concedió un lapso de dos horas a los estudiantes para la lectura del ensayo y la elaboración de la muestra resuntiva. Para evaluar sistemáticamente el corpus textual se diseñaron cuatro matrices (Bolívar, 1999). La primera, usada para analizar la superestructura de las muestras resuntivas (organización textual y función comunicativa de los párrafos); la segunda, recogía los datos relacionados con la macroestructura de las reformulaciones resuntivas por alumno (títulos, subtítulos, ideas principales e ideas secundarias). La tercera matriz registraba el análisis de la microestructura de las muestras resuntivas (progresión informativa, cohesión) y la cuarta matriz estuvo destinada a analizar las macroestrategias resuntivas empleadas por cada alumno (supresión y generalización). El análisis de los resúmenes fue 
vaciado en esta última matriz en atención a los siguientes criterios: la creación de una síntesis adecuada involucraba la selección, literal o parafraseada, de información textual relevante; la creación de una síntesis inadecuada implicaba la selección, literal o parafraseada, de información textual irrelevante o no vinculada al texto fuente y las supresiones consideradas adecuadas serían aquella referidas a la eliminación de información textual no relevante. Una vez culminado el análisis, se procedió a tabular los resultados obtenidos, a los efectos de hacer los cálculos de rigor. La mayor puntuación que era posible obtener se subdividió en cuatro intervalos que dieron origen a cuatro niveles de calidad: Calidad Alta (presencia de introducción, desarrollo y cierre, de ideas principales literales o parafraseadas, descripciones y exposiciones, progresión informativa constante, conectores adaptados al ensayo fuente, entre otros); Calidad Media (presencia de introducción y desarrollo, presencia de algunas ideas principales, literales o parafraseadas, presencia de descripción o exposición, progresión informativa constante, empleo de algunos conectores); Calidad Baja( Presencia de desarrollo solamente, presencia de secuencias textuales ajenas al ensayo fuente, ausencia de muchas ideas principales, empleo inadecuado de conectores y otros elementos cohesivos) y Calidad Deficiente (secuencias textuales ajenas al texto fuente, ausencia de todas las ideas principales, empleo inadecuado de elementos cohesivos lexicales, gramaticales y conectivos). Estos cálculos sirvieron de base para la obtención y análisis de los siguientes resultados:

En primer lugar, los rasgos Macroestructurales de las muestras resuntivas analizadas estuvieron caracterizadas por la omisión de información textual relevante contenida, tanto en los títulos y subtítulos, como en las macroproposiciones del texto fuente (79\%). Esta información fue sustituida por información nueva, ajena al texto. Este hecho parece estar asociado a una visión estandarizada por parte del lector con relación a la distribución de las ideas principales dentro del texto (pensar que se encuentran siempre ubicadas al principio de cada párrafo) y a la carencia de un repertorio léxico abundante que facilite la paráfrasis de las mismas.

En segundo lugar, la conformación superestructural de los resúmenes analizados estuvo constituida, en su mayoría, por el desarrollo como único elemento conformante (75\%), obviando así la introducción y el cierre de los mismos. Este comportamiento 
podría deberse al desconocimiento por parte de los reformuladores sobre los elementos que rigen la organización ideal de un texto, ya que la introducción textual anuncia al lector el tópico y objetivo del texto, mientras que el cierre constituye un recordatorio que sintetiza los aspectos más relevantes del tema tratado (Álvarez, 1996). Todo ello implica la omisión de un porcentaje considerable de ideas principales que no fueron incluidas dentro de las muestras resuntivas. En tercer lugar se evidenció un empleo predominantemente inadecuado de la secuencia textual explicativa (67\%) y argumentativa (100\%), mientras que la descripción se empleó adecuadamente en un 100\% y la ejemplificación se empleó adecuadamente en un 57\%., lo cual evidenció que los resúmenes no reflejaban las secuencias textuales del texto fuente (explicativa y descriptiva) sino que acudieron al uso de otras estrategias típicas del ensayo académico en general (Calsamiglia y Tusón). La argumentación fue empleada para avalar posiciones personales acerca del tema tratado. El patrón de progresión informativa fue preservado (tema constante) y se evidenció un empleo inadecuado de recursos cohesivos gramaticales (referencias distanciadas de sus referentes, sustituciones que no concordaban en género y número con los sujetos, escasas elisiones) y de recursos cohesivos lexicales (inclusión de vocabulario no vinculado a las ideas expresadas en el texto fuente), lo que sugirió nuevamente que las muestras textuales correspondían más a un nuevo texto, que a la reformulación resuntiva del texto original. Con relación al empleo de conectores, se observó el empleo de conectores típicos de los ensayos académicos expositivos (adición, causa-efecto, comparación-contraste, ilustración), sin embargo, su empleo estuvo vinculado a la formulación de aportes personales como argumentos y opiniones ajenos al texto fuente.

Finalmente, con relación al empleo de las macroestrategias de lectura, pudo evidenciarse que la supresión fue empleada de manera arbitraria en las muestras resuntivas, ya que se suprimió información necesaria (83\%) aparentemente por la necesidad de acortar información del ensayo fuente. Esta supresión tampoco respondió a una acción consciente, sino a la exclusión arbitraria de información, desvirtuando la fidelidad del resumen con relación al texto original (67\%) y creando una nueva versión basada en aportes personales de los reformuladores. Las 
generalizaciones, por su parte, no presentaron un orden jerárquico lógico e incorporaban información nueva al texto (56\%), lo que alteró la coherencia de los mismos. Todo ello evidencia que las muestras resuntivas mostraron una calidad baja (58\%), debido a deficiencias observadas en el manejo de recursos cohesivos, estrategias discursivas, preservación de elementos de organización textual y selección de información textual relevante, lo cual permitió observa una coincidencia negativa y positiva significativa (50\%) que permite asociar el inadecuado o adecuado empleo de las macroestrategias de reformulación resuntiva con la producción deficiente o eficiente de resúmenes de ensayos académicos, respectivamente.

\section{CONCLUSIONES}

Desde el punto de vista macroestructural, las muestras resuntivas estuvieron caracterizadas por la omisión de información textual relevante contenida tanto en los títulos y subtítulos como en las macroproposiciones o ideas principales del texto fuente. La estrategia predominante resultó ser la sustitución de esa información por información nueva, ajena al texto y con propósito comunicativo diferente al de la muestra textual originaria.

La superestructura de las muestras resuntivas estuvo conformada predominantemente por la sección del cuerpo o desarrollo textual, omitiéndose en la mayoría elementos introductorios o de cierre. Del mismo modo, la secuencia textual argumentativa predominó en las muestras resuntivas, no habiendo así correspondencia con la secuencia explicativa predominante en el ensayo fuente. Asimismo, en el corpus de reformulaciones analizado destacó el uso de estrategias discursivas de descripción, ejemplificación, explicación, las cuales fueron básicamente empleadas para validar en los textos argumentativos redactados una posición personal y no para presentar información sintetizada acerca del tópico tratado en el ensayo original.

La microestructura de las muestras resuntivas elaboradas mostró empleo deficiente de los recursos cohesivos gramaticales y lexicales. Desde el punto de vista gramatical, en los resúmenes no existía una concordancia adecuada entre referencia y referente. El empleo adecuado de recursos léxicos referidos al vocabulario del ensayo fue 
escaso dentro de las reformulaciones, dando paso más bien al uso de un repertorio léxico propio y nuevo que, aunque se refería al tema del ensayo, no reflejaba fielmente las ideas contenidas en el mismo, por lo que las reformulaciones no eran tales, sino textos personales y nuevos. En las muestras resuntivas se mantuvo el patrón de progresión informativa constante, lo cual sugiere que la muestra reconoció ese patrón y la posibilidad de que por ello haya reconocido el tópico textual. Finalmente, se observó un uso inadecuado de elementos conectivos, bien por establecimiento de relaciones erradas entre proposiciones textuales o porque fueron empleados para reflejar posturas personales y construir una organización secuencial diferente a la del texto original.

Con respecto al uso de las macroestrategias, se evidenció un empleo inadecuado de las mismas. Ello, considerando que las supresiones no perseguían replantear el texto original en sus aspectos más relevantes, sino que resultó ser una supresión de tendencia arbitraria y no consciente por parte de los reformuladores, quienes eliminaron gran cantidad de información textual, tanto relevante como irrelevante, para llevar a cabo la construcción de proposiciones nuevas que reflejaron ideas y posturas personales no provenientes del texto fuente, sino derivadas del tópico que éste planteaba. Ello revela un desconocimiento de la importancia y función de estos procesos para llevar a cabo operaciones de síntesis de información y, por tanto, confirma que la muestra no se encuentra familiarizada con la tarea de resumir.

Por último, se observó una coincidencia significativa, tanto positiva como negativa, entre la baja calidad de los textos producidos y el uso deficiente de las macroestrategias de reformulación resuntiva, que señalan la validez del uso de las macroestrategias en la calidad del resumen. Es decir, la calidad del mismo va a estar relacionada con la calidad en el uso de las macroestrategias. Sin embargo, debido a la naturaleza descriptiva de este trabajo, no se puede afirmar que se trate de una correlación propiamente dicha o de una influencia de una dimensión sobre otra.

Las conclusiones que arroja esta investigación sugiere la necesidad de formar al estudiante y futuro profesional formal y sistemáticamente en cuanto a los rasgos Macroestructurales discursivos propios del ensayo académico, las secuencias textuales y estrategias discursivas de las cuales se nutre, a los fines de que estén 
conscientes de ellas y puedan percibirlas en su proceso de comprensión durante la lectura de tales textos. Además, dentro del proceso de formación del estudiante como lector, debe incluirse explícitamente el uso de las macroestrategias de reformulación resuntiva, toda vez que la elaboración de resúmenes es una de las actividades que con frecuencia se usa para evaluar el conocimiento del alumnado acerca de textos académicos escritos.

Resulta también necesaria la creación de situaciones de aprendizaje alejadas del contexto evaluativo en las que los estudiantes puedan ejercitarse en el conocimiento y empleo de las macroestrategias de reformulación resuntiva, apreciando así su uso como un elemento que contribuirá, no sólo a la mejora de su rendimiento académico, sino también a expandir las posibilidades de comprensión de la información textual que llega a sus manos en cualquier contexto. Esto se traducirá en la optimización de su desempeño como lectores e investigadores. Las actividades que involucren la extracción de información textual implícita y el reconocimiento de la información relevante del texto, como mapas mentales, crucigramas o dameros a partir de la lectura, pirámide de ideas, actividades de reorganización de textos, entre otros, permitirán introducir a los estudiantes a la actividad resuntiva de textos, a través de la identificación de las macroproposiciones textuales y crearán las condiciones idóneas para la posterior elaboración de resúmenes como texto que deriva otra fuente. Finalmente, se recomienda llevar a cabo estudios con el objeto de determinar si existe correlación y/o influencia entre las dimensiones aquí estudiadas, si la aplicación de programas de intervención pedagógica basadas en el modelo macroestratégico permitirían mejorar las habilidades resuntivas de los estudiantes y la calidad macroestructural de sus resúmenes. También resultaría de utilidad explorar si los docentes de diversos niveles educativos están familiarizados con el uso del modelo macroestratégico y si aquellos que hacen uso frecuente del resumen como estrategia didáctica para la evaluación de la comprensión forman conceptual y procedimentalmente a sus estudiantes en este sentido. 


\section{REFERENCIAS CONSULTADAS}

1. Álvarez, T. (1996). El Texto Expositivo-Explicativo: Su Superestructura y Características Textuales. Revista Letras (2008). Vol. 24. Págs. 112-119.

2. Adam, J. (1990). Èléments de Linguistique Textuelle. Liège: Mardaga.

3. Arias, Y. (2009). Correlación entre el Uso de la Estrategia de Predicción y los Niveles de Comprensión del Texto Expositivo. Trabajo de Grado para optar al título de Magister Scientiarum en la Enseñanza de la Lectura y la Escritura. Universidad Nacional Experimental Francisco de Miranda. Coro.

4. Aznar, E., Cross, A. y Quintana, L. (1991). Coherencia Textual y Lectura. Madrid: Ice-Horsori.

5. Arnoux, P.; Noguera, C. y Silvestre, F. (2006). Comprensión Macroestructural y Reformulación Resuntiva de Textos Teóricos. En Cuadernos de Lengua Española. №2. Págs. 25-39. Arco Libros.

6. Bracho, F. (2008). Correlación entre la Evolución de la Lectura Oral y los Niveles de Comprensión del Discurso Académico en Estudiantes de Educación. Trabajo de Grado para optar al título de Magister Scientiarum en la Enseñanza de la Lectura y la Escritura. Universidad Nacional Experimental Francisco de Miranda. Coro.

7. Bolivar, A. (1998). Discurso e Interacción en el Texto Escrito. UCV. Consejo de Desarrollo Científico y Humanístico. Caracas.

8. Calsamiglia, H. y Tusón, A. (1999). Las Cosas del Decir: Manual de Análisis del Discurso. Barcelona: Ariel.

9. DaCosta, F. (2008). Uso de la Nominalización en Textos Académicos Universitarios y el Nivel de Comprensión Alcanzado por los Estudiantes al Leer los Referidos Textos. Trabajo de Grado para optar al título de Magister Scientiarum en la Enseñanza de la Lectura y la Escritura. Universidad Nacional Experimental Francisco de Miranda. Coro.

10. Fraca, L. (2001). El Resumen como Texto: Un Abordaje Evolutivo. En Cuadernos de Lengua y Habla. №3. (2001-2002). Págs. 293-306.

11. Fumero, F. (1997). El Ensayo como Tipo de Texto. Caracas: FUDUPEL

12. García de Díaz, M. (2008). La Comprensión Inferencial del Discurso Académico. Trabajo Mimeografiado presentado en el Doctorado en Estudios del Discurso. Universidad Central de Venezuela.

13. García, J. (2006). Lectura y Conocimiento. Barcelona: Paidós. 
14. Hernández, R.; Fernández, C. y Baptista, L. (1998). Metodología de la Investigación. México: McGraw-Hill.

15. Jordán, C. (2009). El Andamiaje Instruccional en la Comprensión Inferencial del Texto Expositivo en Estudiantes de Educación Básica. Trabajo de Grado para optar al título de Magister Scientiarum en la Enseñanza de la Lectura y la Escritura. Universidad Nacional Experimental Francisco de Miranda. Coro.

16. Kintsch, W. y van Dijk, T. (1983). Strategies on Discourse Comprehension. New York: Academic Press.

17. León, J. (1996). La Comprensión y Recuerdo de Textos Expositivos a través del Análisis de algunas Variables del Texto y Lector. En Infancia y Aprendizaje (1998). Vól.56. Págs. 51-76.

18. Smith, F. (1985). Comprensión de la Lectura. México, D.F.: Trillas.

19. Richards, J.; Platt, J. y Platt, H. (1992). Longman Dictionary of Language Teaching and Applied Linguistics. Malaysia: Longman.

20. Ruiz, C. (2002). Instrumentos e Investigación Educativa. Barquisimeto: CIDEG.

\section{REFERENCES CONSULTED}

1. Álvarez, T. (1996). The Expository-Explanatory Text: Its Superstructure and Textual Characteristics. Letters Magazine (2008). Vol. 24. Pages. 112-119.

2. Adam, J. (1990). Èléments de Linguistique Textuelle. Liège: Mardaga.

3. Arias, Y. (2009). Correlation between the Use of the Prediction Strategy and the Levels of Understanding the Expository Text. Degree work to apply for the title of Magister Scientiarum in the Teaching of Reading and Writing. Francisco de Miranda National Experimental University. Chorus.

4. Aznar, E., Cross, A. and Quintana, L. (1991). Textual Consistency and Reading. Madrid: Ice-Horsori.

5. Arnoux, P.; Noguera, C. and Silvestre, F. (2006). Macrostructural Comprehension and Restructive Reformulation of Theoretical Texts. In Spanish Language Notebooks. №2. P. 25-39. Arch Books.

6. Bracho, F. (2008). Correlation between the Evolution of Oral Reading and the Levels of Understanding Academic Speech in Education Students. Degree work 
to apply for the title of Magister Scientiarum in the Teaching of Reading and Writing. Francisco de Miranda National Experimental University. Chorus.

7. Bolivar, A. (1998). Speech and Interaction in Written Text. UCV. Council of Scientific and Humanistic Development. Caracas.

8. Calsamiglia, H. and Tusón, A. (1999). The Things of Saying: Handbook of Speech Analysis. Barcelona: Ariel.

9. DaCosta, F. (2008). Use of Nominalization in University Academic Texts and the Level of Comprehension Achieved by Students when Reading the Referred Texts. Degree work to apply for the title of Magister Scientiarum in the Teaching of Reading and Writing. Francisco de Miranda National Experimental University. Chorus.

10. Fraca, L. (2001). Summary as Text: An Evolutionary Approach. In Language and Speech Notebooks. №3. (2001-2002). P. 293-306.

11. Fumero, F. (1997). The Essay as Type of Text. Caracas: FUDUPEL

12. García de Díaz, M. (2008). The Inferential Understanding of Academic Speech. Mimeographed work presented at the Doctorate in Speech Studies. Central University of Venezuela.

13. García, J. (2006). Reading and knowledge. Barcelona: Paidós.

14. Hernández, R.; Fernández, C. and Baptista, L. (1998). Investigation methodology. Mexico: McGraw-Hill.

15. Jordan, C. (2009). Instructional Scaffolding in Inferential Understanding of Expository Text in Basic Education Students. Degree work to apply for the title of Magister Scientiarum in the Teaching of Reading and Writing. Francisco de Miranda National Experimental University. Chorus.

16. Kintsch, W. and van Dijk, T. (1983). Strategies on Discourse Comprehension. New York: Academic Press.

17.León, J. (1996). The Understanding and Memory of Expository Texts through the Analysis of some Variables of the Text and Reader. In Children and Learning (1998). Vol. 56. P. 51-76.

18. Smith, F. (1985). Reading comprehension. Mexico, D.F.: Trillas.

19. Richards, J.; Platt, J. and Platt, H. (1992). Longman Dictionary of Language Teaching and Applied Linguistics. Malaysia: Longman. 
EPISTEME KOINONIA

Revista Electrónica de Ciencias de la Educación, Humanidades, Artes y Bellas Artes

Año I. Vol I. N 1 .

Enero - Junio, 2018

Hecho el depósito de Ley: FA2018000022

FUNDACIÓN KOINONIA (F.K).

Santa Ana de Coro, Venezuela

Yovaida Dovale

20. Ruiz, C. (2002). Instruments and Educational Research. Barquisimeto: CIDEG.

C2018 por los autores. Este artículo es de acceso abierto y distribuido según los términos y condiciones de la licencia Creative Commons Atribución-NoComercial-Compartirlgual 4.0 Internacional (CC BY-NC-SA 4.0)

(https://creativecommons.org/licenses/by-nc-sa/4.0/). 